\title{
Analytical study of the horizontal ducting of sound by an oceanic front over a slope
}

\author{
Ying-Tsong Lin a) and James F. Lynch \\ Applied Ocean Physics and Engineering Department, Woods Hole Oceanographic Institution, \\ Woods Hole, Massachusetts 02543 \\ ytlin@whoi.edu,jlynch@whoi.edu
}

\begin{abstract}
The horizontal ducting of sound by an oceanic temperature front over a sloping bottom is studied with an idealized wedge model consisting of a lateral interface across the slope. The water outside the frontal interface has higher temperature, hence faster sound speed, and it will produce inshore reflection/refraction of the sound. Combining the offshore refraction caused by the sloping bottom, propagating sound can be ducted along the front. An analytical solution to the sound pressure field in the idealized model is derived, and an example is presented to demonstrate and discuss the ducting effect.
\end{abstract}

(C) 2012 Acoustical Society of America

PACS numbers: 43.30.Bp, 43.20.Bi, 43.20.Mv [WS]

Date Received: September 20,2011 Date Accepted: October 27, 2011

\section{Introduction}

The horizontal refraction of sound over a slope by the bathymetry has been studied extensively in the past decades (see a recent study by Heaney and Murray ${ }^{1}$ and references therein). The effect of sound speed fronts on sound refraction has also been investigated by Katsnelson et $a l^{2}$ and Badiey et $a l^{3}{ }^{3}$ for the cases where sound speed fronts are formed by either shelfbreak fronts or nonlinear internal waves. This paper is focused on the horizontal ducting effect caused jointly by these two environmental factors, which was initially noted by Lynch et al. ${ }^{4}$

A simplified environmental model is considered so that an analytical solution to the sound pressure field can be sought. This model is an idealized wedge bounded by a horizontal pressure-release surface and an impenetrable slope with an angle $\alpha$, and contains a lateral/frontal interface (see Fig. 1). The lateral interface is placed opposite to the wedge apex and separates two different sound speed regions: $c_{1}$ between the apex and the interface, and $c_{2}$ outside the interface. The sound speed outside the frontal interface is taken to be greater $\left(c_{2}>c_{1}\right)$, hence sound impinging on the interface from inside can reflect back. A cylindrical coordinate system $(r, \theta, y)$ is used here; $r$ is the radial distance from the wedge apex, $\theta$ is the clockwise angle from the surface, and $y$ is the horizontal distance in the direction parallel to the wedge apex. The medium density $\rho$ in the model is considered to be unity as is appropriate for water.

It is worth noting two major simplifications made for the bottom boundary and the frontal interface in the model. In reality, underwater sound can penetrate into the bottom, and propagation of higher-order vertical modes with a greater grazing angle to the bottom suffers from significant attenuation. In other words, the impenetrable model employed here can only provide a good approximation to the lower-order vertical modes. The other notable simplification assumes an infinite sound speed gradient at the front (a sharp interface), which differs from the real, finite-gradient fronts observed in the ocean. Thus the cycle distance of horizontal ducting modes found in the model will be shorter comparing to realistic cases. Discussion of other model simplifications will be provided later in the paper.

\footnotetext{
${ }^{\text {a) }}$ Author to whom correspondence should be addressed.
} 


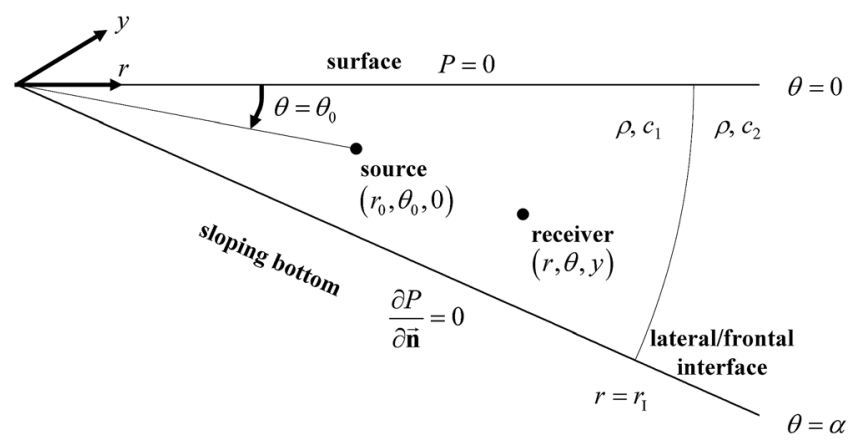

Fig. 1. Schematic diagram of an idealized wedge with a lateral interface. The surface is a pressure-release boundary, and the slope angle of the impenetrable bottom is $\alpha$. The lateral interface is placed at $r=r_{\mathrm{I}}$ to separate two different sound speed regions.

\section{Analytical solution}

Derivation of an analytical solution to the sound field in the simplified model closely follows a combined approach used by Frisk ${ }^{5}$ for a similar wedge problem without the lateral interface. This approach includes wavenumber integration, an eigenfunction expansion, and the endpoint method. A complex integration technique with Cauchy's integral theorem is also utilized in this study.

The sound pressure field $P(r, \theta, y)$ due to a point source located at $\left(r_{0}, \theta_{0}, 0\right)$ and emitting continuous sound waves at a single frequency $f$ can be determined from the horizontal wavenumber integral

$$
P(r, \theta, y)=\frac{1}{2 \pi} \int_{-\infty}^{\infty} G\left(r, \theta, k_{y}\right) e^{i k_{y} y} d k_{y}
$$

where $k_{y}$ is the horizontal wavenumber along $y$, and $G$ is the wavenumber spectrum governed by the following equation:

$$
\frac{1}{r} \frac{\partial}{\partial r}\left[r \frac{\partial G}{\partial r}\right]+\frac{1}{r^{2}} \frac{\partial^{2} G}{\partial \theta^{2}}+\left(k^{2}-k_{y}^{2}\right) G=-4 \pi \frac{\delta\left(r-r_{0}\right)}{r} \delta\left(\theta-\theta_{0}\right),
$$

where the medium wavenumber $k$ is $k_{1}=2 \pi f / c_{1}$ for $r<r_{\mathrm{I}}$ and $k_{2}=2 \pi f / c_{2}$ for $r>r_{\mathrm{I}}$. One can employ an eigenfunction expansion and Sturm-Liouville theory to solve Eq. (2). As shown by Frisk, ${ }^{5}$ the expansion of $G$ is formed by the angular eigenfunctions/modes:

$$
G\left(r, \theta, k_{y}\right)=\sum_{n=1}^{\infty} \Phi_{n}\left(\theta_{0}\right) A_{n}\left(r, k_{y}\right) \Phi_{n}(\theta)
$$

where $n$ is the order of the angular mode $\Phi_{n}(\theta)$, and $\Phi_{n}(\theta)=\sqrt{2 / \alpha} \sin \left(\eta_{n} \theta\right)$ with the eigenvalue $\eta_{n}=(n-1 / 2) \pi / \alpha$. Because the medium wavenumber $k$ is only a function of $r$, separation of variables can be completed. Also, because the boundary condition in $\theta$ is either Dirichlet- or Neumann-type, the angular modes satisfy a proper SturmLiouville equation and, therefore, constitute a complete and orthonormal set for expanding an arbitrary function.

To determine the amplitude of the $n$th angular mode, $A_{n}\left(r, k_{v}\right)$, we substitute the expansion of $G$ into Eq. (2) and carry out the separation of variables to obtain the following ordinary differential equation for $A_{n}\left(r, k_{y}\right)$ : 


$$
\frac{1}{r} \frac{d}{d r}\left[r \frac{d A_{n}\left(r, k_{y}\right)}{d r}\right]+\left(k^{2}-k_{y}^{2}-\frac{\eta_{n}^{2}}{r^{2}}\right) A_{n}\left(r, k_{y}\right)=-4 \pi \frac{\delta\left(r-r_{0}\right)}{r} .
$$

Besides the boundary conditions at $r=0$ and $r=\infty$, i.e., $A_{n}(0)=0$ and $A_{n}(\infty)$ satisfies the Sommerfeld radiation boundary condition, there are two interface conditions enforced at $r=r_{\mathrm{I}}$ for the continuity of pressure and normal displacement, i.e., $A_{n}\left(r_{\mathrm{I}}^{-}\right)=A_{n}\left(r_{\mathrm{I}}^{+}\right)$and $\mathrm{d} A_{n}\left(r_{\mathrm{I}}^{-}\right) / \mathrm{d} r=\mathrm{d} A_{n}\left(r_{\mathrm{I}}^{+}\right) / \mathrm{d} r$.

Considering the source is located between the apex and the front, $0<r_{0}<r_{\mathrm{I}}$, the endpoint method can be employed to solve for $A_{n}$ with two homogeneous solutions separately satisfying the boundary condition at $r=0$ or the interface conditions at $r=r_{\mathrm{I}}$. The endpoint method is a standard approach for solving a boundary value problem, and the detailed description can be found in Ref. 5. Because Eq. (4) is a Bessel's differential equation, the two homogenous solutions and $A_{n}^{(0)}\left(r, k_{y}\right)=J_{\eta_{n}}\left(k_{r 1} r\right)$ and $A_{n}^{(1)}\left(r, k_{y}\right)=H_{\eta_{n}}^{(1)}\left(k_{r 1} r\right)+R_{n} \cdot H_{\eta_{n}}^{(2)}\left(k_{r 1} r\right)$. Their Wronskian is defined as $W\left[A_{n}^{(0)}\left(r_{0}\right), A_{n}^{(1)}\left(r_{0}\right)\right]=i 2\left(1-R_{n}\right) /\left(\pi k_{r 1} r_{0}\right)$, and it is used for determining the denominator of the final solution of Eq. (4). Because the Hankel functions of the first and second kinds in the second homogeneous solution indicate outgoing and incoming waves respectively, the function $R_{n}$ is the reflection coefficient at the interface. With straightforward, but lengthily, manipulations, the reflection coefficient can be derived:

$$
R_{n}=-\frac{k_{r 1} H_{\eta_{n}+1}^{(1)}\left(k_{r 1} r_{\mathrm{I}}\right) H_{\eta_{n}}^{(1)}\left(k_{r 2} r_{\mathrm{I}}\right)-k_{r 2} H_{\eta_{n}}^{(1)}\left(k_{r 1} r_{\mathrm{I}}\right) H_{\eta_{n}+1}^{(1)}\left(k_{r 2} r_{\mathrm{I}}\right)}{k_{r 1} H_{\eta_{n}+1}^{(2)}\left(k_{r 1} r_{\mathrm{I}}\right) H_{\eta_{n}}^{(1)}\left(k_{r 2} r_{\mathrm{I}}\right)-k_{r 2} H_{\eta_{n}}^{(2)}\left(k_{r 1} r_{\mathrm{I}}\right) H_{\eta_{n}+1}^{(1)}\left(k_{r 2} r_{\mathrm{I}}\right)}
$$

where $k_{r 1}=\sqrt{k_{1}^{2}-k_{y}^{2}}, k_{r 2}=\sqrt{k_{2}^{2}-k_{y}^{2}}$, and the recurrence relation of Hankel functions, ${ }^{6} d H_{v}^{(1,2)}\left(k_{0} r\right) / d r=-k_{0} H_{v+1}^{(1,2)}\left(k_{0} r\right)+H_{v}^{(1,2)}\left(k_{0} r\right) v / r$, is utilized. Following the steps of the endpoint method ${ }^{5}$ and using the two homogeneous solutions presented in the preceding text and their Wronskian, we can derive

$$
A_{n}\left(r ; k_{y}\right)= \begin{cases}i 2 \pi^{2} \frac{J_{\eta_{n}}\left(k_{r 1} r\right)\left[H_{\eta_{n}}^{(1)}\left(k_{r 1} r_{0}\right)+R_{n} H_{\eta_{n}}^{(2)}\left(k_{r 1} r_{0}\right)\right]}{1-R_{n}} & \text { for } \quad r_{0} \geq r \geq 0, \\ i 2 \pi^{2} \frac{J_{\eta_{n}}\left(k_{r 1} r_{0}\right)\left[H_{\eta_{n}}^{(1)}\left(k_{r 1} r\right)+R_{n} H_{\eta_{n}}^{(2)}\left(k_{r 1} r\right)\right]}{1-R_{n}} & \text { for } \quad r_{\mathrm{I}} \geq r \geq r_{0} .\end{cases}
$$

Because the arguments of the Hankel functions in $A_{n}\left(r, k_{y}\right), k_{r 1}$ and $k_{r 2}$, result from complex square roots, the function $A_{n}$ has two Riemann sheets on the complex $k_{y}$ plane. To integrate $A_{n}$, we need to apply branch cuts to enable an integral path to move from one Riemann sheet to the other. The Pekeris cut ${ }^{7}$ is preferred here because it can expose all of the singularities, which occur at $R_{n}=1$. The branch points of the Pekeris cut are at $k_{y}=+k_{2}$ and $-k_{2}$, and the cuts start there and extend to $k_{2}+i \infty$ and $-\left(k_{2}+i \infty\right)$, respectively.

Substituting $A_{n}$ and $\Phi_{n}$ in Eq. (3) and carrying out the wavenumber integration Eq. (1) will give the solution for the sound field, i.e.,

$$
P(r, \theta, y)=\frac{1}{\alpha \pi} \sum_{n}\left[\sin \left(\eta_{n} \theta_{0}\right) \sin \left(\eta_{n} \theta\right) \int_{-\infty}^{\infty} A_{n}\left(r ; k_{y}\right) e^{i k_{y} y} d k_{y}\right] .
$$

The integration solution of Eq. (7) is formally the full solution to the problem, and one can use a numerical wavenumber integration technique ${ }^{8}$ to calculate its values. Here a complex integration technique used in normal mode theory ${ }^{8}$ is adopted for 
calculating the wavenumber integration, and the resultant normal mode solution will be in a form of summations and readily provide physical insight to the horizontal ducting of sound in the waveguide.

According to Cauchy's integral theorem, a complex contour integral along a closed path equals to the sum of the residues of the integrand at its singularities inside the closed domain. Hence the wavenumber integral along the real $k_{y}$ axis in Eq. (7) can be determined from the sum of the residues minus the line integral along a half circle passing around the branch cut in the upper complex $k_{y}$ plane. As long as $y$ is not zero, the integrand $A_{n}\left(r, k_{y}\right) \exp \left(i k_{y} y\right)$ vanishes when $k_{y}$ goes to infinity in the upper complex plane. Therefore making the radius of the half-circle path infinite can reduce the line integral to be only around the branch cut. Also, if we are only interested in the far field, the branch line integral can be further neglected. Then the wavenumber integral is simply determined from the sum of the residues, i.e.,

$$
\int_{-\infty}^{\infty} A_{n}\left(r, k_{y}\right) e^{i k_{y} y} d k_{y} \cong-4 \pi^{3} \sum_{m} \frac{J_{\eta_{n}}\left(k_{r 1}^{n m} r_{0}\right) J_{\eta_{n}}\left(k_{r 1}^{n m} r\right)}{d\left(1-R_{n}\right) /\left.d k_{y}\right|_{k_{y}=k_{n m}}} e^{i k_{n m} y} \quad \text { for } y \neq 0,
$$

where $k_{n m}$ is the $m$ th singularity of the integrand $A_{n}\left(r, k_{y}\right) \exp \left(i k_{y} y\right)$, and it can be determined from $R_{n}=1$ where the zeros of the denominator of $A_{n}$ occur, as shown in Eq. (6). Also, because the Pekeris branch cut is chosen for the complex integration, all of the singularities are exposed, so both the propagating modes and the leaky modes will be included in the solution. ${ }^{7,8}$ Substituting the wavenumber integral in Eq. (7) yields the final solution to the sound field due to a point source located between the apex and the front:

$$
P(r, \theta, y)=\frac{4 \pi^{2}}{\alpha} \sum_{n} \sum_{m} Q_{n m}\left(r_{0}, \theta_{0}\right) \sin \left(\eta_{n} \theta\right) \Psi_{n m}\left(k_{n m}, r\right) e^{i k_{n m} y},
$$

where $0<r_{0}<r_{\mathrm{I}}, k_{r 1}^{n m}=\sqrt{k_{1}^{2}-k_{n m}^{2}}, k_{r 2}^{n m}=\sqrt{k_{2}^{2}-k_{n m}^{2}}$,

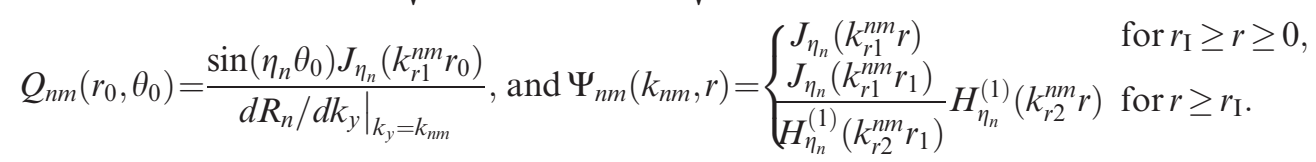

The last solution for the sound field at $r \geq r_{\mathrm{I}}$ is obtained by matching the interface conditions.

Note that the angular eigenvalue $\eta_{n}$ determines the order of the Bessel and Hankel functions. Also, $\Psi_{n m}$ constitutes another set of orthogonal modes for the radial component of the sound field, and these radial modes can propagate across the slope in the $y$ direction with the modal wavenumber $k_{n m}$. Analogous to the whispering gallery effect ${ }^{9}$ under a dome or near a circular wall, the radial modes seen here are also expressed by cylinder functions. When the wavenumber $k_{n m}$ is purely real or has only an insignificant imaginary value, the corresponding radial mode is specified to be an "oceanic whispering gallery" mode, and it will propagate along the frontal "wall" with very little energy radiating outwards in the radial direction.

\section{Numerical example}

An example of sound propagation in the idealized wedge is presented. The slope angle $\alpha$ is $3 \mathrm{deg}$, which is a typical angle for a continental slope. The front is located at $r_{\mathrm{I}}=4 \mathrm{~km}$, and the sound speed is $1500 \mathrm{~m} / \mathrm{s}\left(c_{1}\right)$ inside and $1520 \mathrm{~m} / \mathrm{s}\left(c_{2}\right)$ outside. The acoustic frequency considered here is $25 \mathrm{~Hz}$.

The Newton-Raphson method is utilized to obtain the radial eigenvalues $k_{n m}$ by solving the characteristic equation $R_{n}=1$. The eigenvalues are shown in Fig. 2(a) for a range of angular order $n$ from 1 to 3 . One can see that some of the radial modes 
(a) Eigenvalues $k_{n m}$ of radial modes

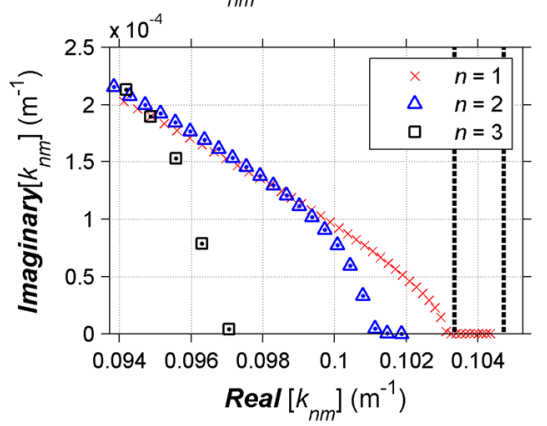

(c) $n=2$

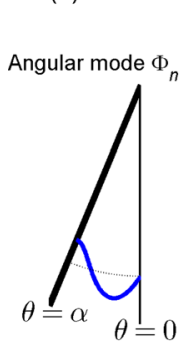

Radial modes $\Psi_{n m}$

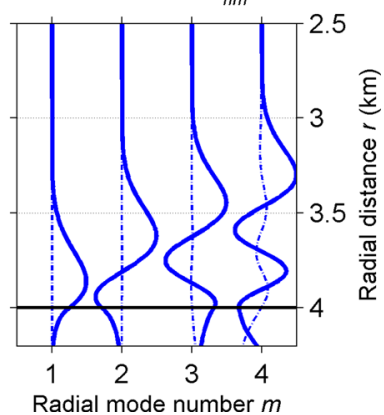

(b) $n=1$

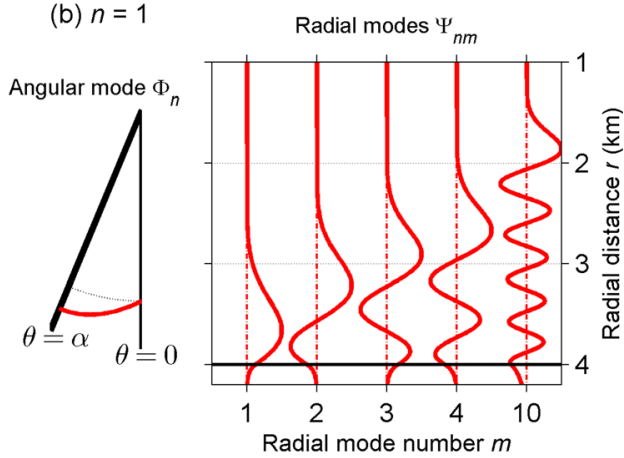

(d) $n=3$

Radial modes $\Psi_{n m}$

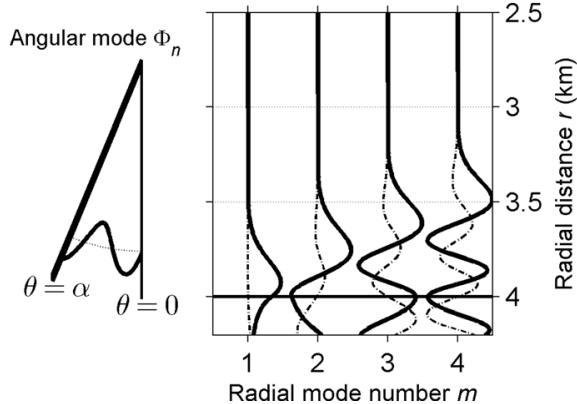

Fig. 2. (Color online) Eigenfunctions of the sound field in the example problem. (a) shows the distribution of the radial eigenvalues $k_{n m}$ for different angular orders, where $n=1$ to 3 , and the angular eigenvalue $\eta_{n}=$ $(n-1 / 2) \pi / \alpha$, where $\alpha=3 \mathrm{deg}$. Curves in (b) to (d) are the mode functions of the first three angular orders, and the imaginary part is shown in the dash-dotted curves.

have a very small, or even vanishing, imaginary part in their eigenvalues. These radial modes are the whispering gallery modes, and they can propagate across the slope with very little modal attenuation. In the current example, there are 10 whispering gallery modes for the first angular order, and the corresponding mode functions are (partly) shown in Fig. 2(b). One can see that the turning point of the radial mode gradually approaches the apex as the mode number $m$ increases. This is because higher-order radial modes have greater horizontal grazing angles, and so they can propagate further into the wedge.

As the angular order goes higher, fewer and fewer whispering gallery modes exist, see Fig. 2(a) for $n=2$ and 3. This is because higher-order angular modes have greater vertical grazing angles, and so a smaller horizontal angular aperture is allowed for the associated sound ray impinging at the front below the critical angle for total internal reflection. Also because the angular modes with greater vertical grazing angles have stronger horizontal refraction from the sloping bottom, the turning point of the radial mode with higher angular order goes less into the wedge, as shown in Figs. 2(c) and 2(d). Geometrical similarity is observed in the radial mode functions, and for those not considered to be whispering gallery modes, they are the leaky modes with a significant imaginary part and describe the radiation of the sound outwards in the radial direction.

A $0-\mathrm{dB}$ (unity source level), $25-\mathrm{Hz}$ point source is placed at $\theta=1.5 \mathrm{deg}$ (half of the slope angle), and Fig. 3 shows the sound pressure on the source plane in $r$ and $y$ when the source is just $100 \mathrm{~m}$ away from the front. The whispering gallery effect along the front can be seen in the angular mode intensity, Figs. 3(a) to 3(c). The dependency of the turning point on the angular order $n$ is also observed. In addition, one can see that the cycle distance of the ducted sound decreases as the angular order increases. 
(a) Angular mode 1 intensity

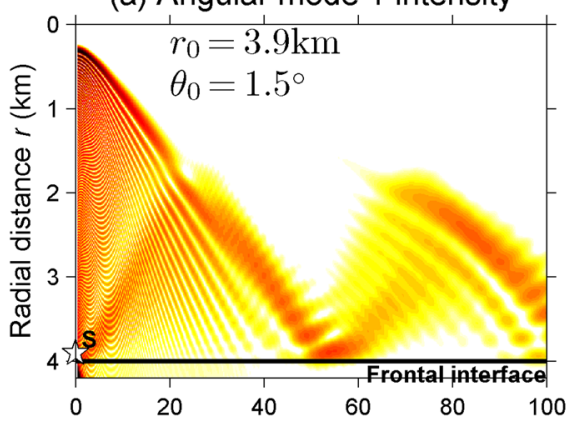

(c) Angular mode 3 intensity

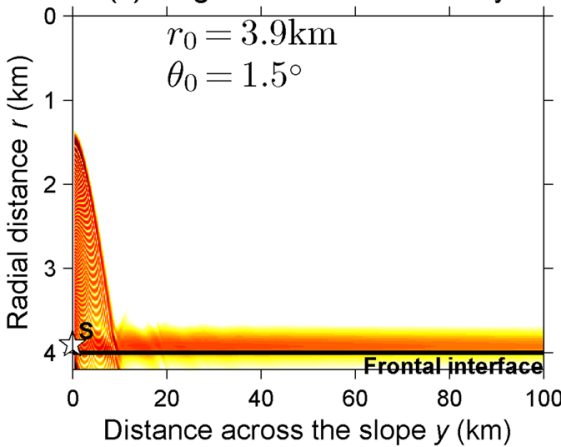

(b) Angular mode 2 intensity

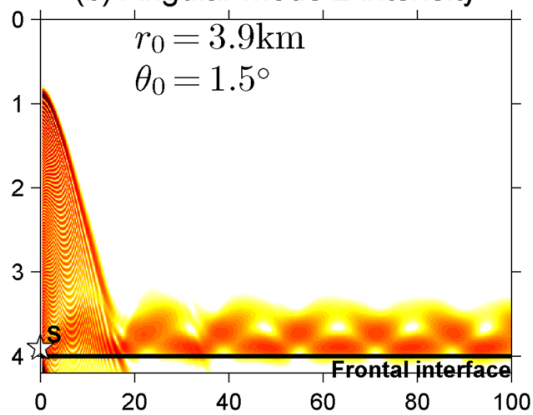

(d) Total sound intensity

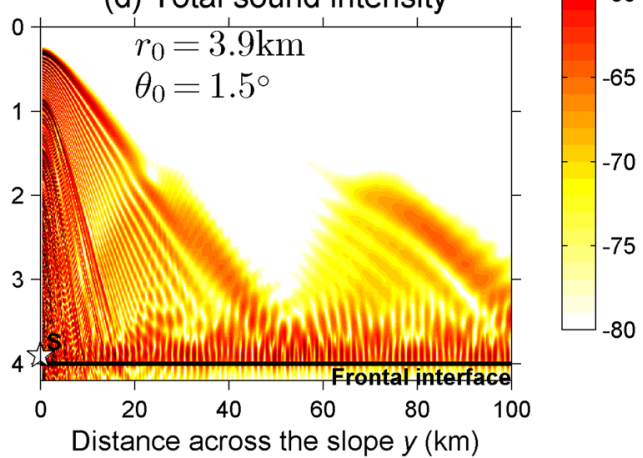

Fig. 3. (Color online) Sound pressure on the source plane in $r$ and $y$ in the example problem. The source is located at $r_{0}=3.9 \mathrm{~km}$ and $\theta_{0}=1.5^{\circ}$, and the source level is $0 \mathrm{~dB}$. (a) to (c) show the intensity of the first three angular modes, and (d) is the total sound intensity with contributions from five angular modes. Note that angular modes 4 and 5 do not have whispering gallery modes.

This can be confirmed by a direct calculation with the eigenvalues of radial modes via the following formulation,

$$
L_{n m}=\frac{2 \pi}{k_{m}-k_{n, m+1}},
$$

where $L_{n m}$ is the cycle distance of the modal interference between $\Psi_{n m}$ and $\Psi_{n, m+1}$. The total sound intensity with contributions from five angular modes is shown in Fig. 3 (d), and more complicated modal interference between different angular orders can be seen.

A multimedia movie file, Mm. 1, shows the sound pressure field for different source positions. As the source moves away from the front, fewer and fewer oceanic whispering gallery modes are excited. Also, for each angular mode, once the source passes its greatest turning point, it will not be ducted by the front, and we will only see the mode radiating outwards as if there was no front.

Mm. 1. Sound pressure field excited by a point source at different locations. This is a file of type "mpg" (1.9 Mb).

\section{Conclusion}

The horizontal ducting of sound by a front with faster sound speed over a slope is studied analytically via an idealized model. The example provided here clearly shows that the front can provide an oceanic whispering gallery condition. Excitation of such whispering galley modes depends on the number of the vertical mode, which is the angular mode in this study as the $\theta$ axis is in the vertical direction. Higher order 
vertical modes with greater vertical grazing angles have less potential for whispering gallery ducting, but their corresponding whispering gallery modes have much shorter cycle distances across the slope with turning points closer to the front.

The acoustic frequency in the calculation example is chosen to be $25 \mathrm{~Hz}$ to keep the simplicity of modal structure for analysis. Because the Hankel-function solutions are scaled by the acoustic wavelength, the results from the example can be extended to higher frequency cases. When the number of the modes is large, a ray approach is more appropriate and should be used for computing the sound field.

Besides the simplifications made for the seafloor and the frontal interface, other environmental variability seen in shelfbreak and slope areas are also not considered in this study. These include the water column inhomogeneity caused by the frontal dynamics, and the irregularity and roughness of the frontal interface due to the meandering and intrusions of shelfbreak fronts. Numerical models of three-dimensional sound propagation are required for considering more realistic frontal conditions, but the theoretical analysis shown in this paper can provide first-order physical insight to the problem.

\section{Acknowledgments}

This work was sponsored by the Office of Naval Research under Grant No. N00014-10-10040. The authors also thank Arthur Newhall of the Woods Hole Oceanographic Institution for his editorial assistance with the manuscript.

\section{References and links}

${ }^{1}$ K. D. Heaney and J. J. Murray, "Measurement of three-dimensional propagation in a continental shelf environment," J. Acoust. Soc. Am. 125, 1394-1402 (2009).

${ }^{2}$ B. G. Katsnelson, J. Lynch, and A. V. Tshoidze, "Space-frequency distribution of sound field intensity in the vicinity of the temperature front in shallow water," Acoust. Phys. 53, 611-617 (2007).

${ }^{3}$ M. Badiey, B. G. Katsnelson, Y.-T. Lin, and J. F. Lynch, "Acoustic multipath arrivals in the horizontal plane due to approaching nonlinear internal waves," J. Acoust. Soc. Am. 129, EL141-EL147 (2011).

${ }^{4}$ J. F. Lynch, J. A. Colosi, G. G. Gawarkiewicz, T. F. Duda, A. D. Pierce, M. Badiey, B. G. Katsnelson, J. E. Miller, W. Siegmann, C.-S. Chiu, and A. Newhall, "Consideration of fine-scale coastal oceanography and 3-D acoustics effects for the ESME sound exposure model," IEEE J. Ocean. Eng. 31, 33-48 (2006).

${ }^{5}$ G. V. Frisk, Ocean and Seabed Acoustics: A Theory of Wave Propagation (Prentice-Hall, Englewood Cliffs, NJ, 2007), pp. 275-280.

${ }^{6}$ M. Abramowitz and I. A. Stegun, Handbook of Mathematical Functions: With Formulas, Graphs, and Mathematical Tables (National Bureau of Standards Applied Mathematics Series 55, Washington, DC, 1964), pp. 361-361.

${ }^{7}$ C. L. Pekeris, "Theory of propagation of explosive sound in shallow water," in Propagation of Sound in the Ocean, edited by M. Ewing, J. L. Worzel, and C. L. Pekeris, Geol. Soc. Am. Mem. 27 (Geological Society of America, New York, 1948).

${ }^{8}$ F. B. Jensen, W. A. Kuperman, M. B. Porter, and H. Schmidt, Computational Ocean Acoustics (AIP, Woodbury, NY, 1994), Chaps. 4 and 5.

${ }^{9}$ L. Rayleigh, "The problem of the whispering gallery," Philos. Mag. 20, 1001-1004 (1910). 